\title{
A comparison of screw versus drill and curettage epiphysiodesis to correct leg-length discrepancy
}
M. Troy
B. Shore
P. Miller
S. Mahan
D. Hedequist
B. Heyworth
J. Kasser
S. Spencer
M. Glotzbecker

\begin{abstract}
Purpose To compare two common surgical techniques of epiphysiodesis: drill/curettage epiphysiodesis (PDED) versus cross screw epiphysiodesis (PETS). The hypothesis is that the two techniques have similar efficacy but demonstrate differences in length of hospital stay (LOS), time to return to activity and complication rates.
\end{abstract}

Methods A retrospective review of growing children and adolescents less than 18 years old who required an epiphysiodesis with leg-length discrepancy (LLD) of $2 \mathrm{~cm}$ to $6 \mathrm{~cm}$ with minimum two years of follow-up was conducted. Characteristics including age at surgery, gender, epiphysiodesis location, side, operative time, LOS and hardware removal were compared across treatment groups. LLD, expected growth remaining (EGR) and bone age were determined preoperatively and at most-recent visit. The correction ratio (change in EGR) was calculated along with a $95 \%$ confidence interval $(\mathrm{Cl})$ to assess if correction in leg length was achieved.

Results A total of 115 patients underwent epiphysiodesis in the femur (53\%), tibia (24\%) or a combination (24\%). The cohort was $47 \%$ male, with a mean age of 12.6 years ( 7.7 to 17.7 ) at surgery. Median follow-up was 3.7 years (2.0 to 12.7). In all, 23 patients underwent PETS and 92 patients had PDED. Both treatment groups achieved expected LLD correction. There was no significant difference in median operative time, complication rates or LOS. PETS patients returned to activity at a mean 1.4 months (interquartile range (IQR) 0.7

Department of Orthopaedic Surgery, Boston Children's Hospital, Boston, Massachusetts, USA

Correspondence should be sent to M. Glotzbecker, 300 Longwood Ave, Hunnewell 2, Boston, Massachusetts 02115, USA.

E-mail: Michael.Glotzbecker@childrens.harvard.edu to 2.1) while PDED patients returned at a mean 2.4 months (IQR 1.7 to 3$)(p<0.001)$.

Conclusion Effectiveness in achieving expected correction, LOS and operative time are similar between screw and drill/ curettage epiphysiodesis. Patients undergoing PETS demonstrated a faster return to baseline activity than patients with PDED.

Level of Evidence: III

Cite this article: Troy M, Shore B, Miller P, Mahan S, Hedequist D, Heyworth B, Kasser J, Spencer S, Glotzbecker M. A comparison of screw versus drill and curettage epiphysiodesis to correct leg-length discrepancy. J Child Orthop 2018;12:509-514. DOI: 10.1302/1863-2548.12.180030

Keywords: epiphysiodesis; growth arrest; transphyseal screws; drill and curettage; leg-length discrepancy

\section{Introduction}

Epiphysiodesis is often performed in order to correct leglength discrepancy (LLD). Phemister ${ }^{1}$ first described an open procedure in 1933, destroying the growth plates on a permanent basis between the epiphysis and metaphysis through excised windows on both condyles. Canale and Christian $^{2}$ depicted a drill and curettage technique to destroy either the distal femoral physis, proximal tibial physis or both physes. This technique provided potential leg length equalization, but on a permanent basis.

Percutaneous epiphysiodesis using transphyseal screws (PETS) has also been widely adopted in addition to Canale's method. Métaizeau et $\mathrm{al}^{3}$ described this procedure in 1998, using 7.3-mm cannulated screws across the distal femoral or proximal tibial physis. Proponents of PETS advocate that the method offers a simple technique, short operating time, decreased hospitalization, low complication rate and the reliable reversibility and recovery of growth as described by Anderson et al and Haas in a rabbit model. ${ }^{3-9}$ However, the debate of optimal surgical technique remains unanswered. Niedzielski et $\mathrm{al}^{10}$ and Ramseier et $\mathrm{al}^{11}$ both retrospectively reviewed small cohorts of patients (34 and 11 respectively) with full percutaneous drill/curettage epiphysiodesis (PDED) procedures for treatment of LLD, while denoting the relative simplicity, short hospitalization and low complication rate of the procedure. Babu et al ${ }^{12}$ promotes the use of the Canale technique due to a lower 
complication rate, while Campens et $a^{13}$ depicts equal efficiency and complication rates, yet lower operative time when using the PETS technique. Others also argue the true reversibility of the PETS procedure due to potential physis violation. ${ }^{14,15}$ Remaining techniques such as Blount stapling, eight-plate epiphysiodesis for angular deformity and tension banding remain in use, yet are unexamined within the confines of this study.

The purpose of this study is to compare two common surgical techniques of epiphysiodesis: PDED versus PETS. The study hypothesis is that the two techniques will have similar efficacy in correcting LLD but demonstrate differences in length of hospital stay (LOS), time to return to activity and postoperative complication rate.

\section{Methods}

This retrospective study was approved by the Institutional Review Board. Medical records between 2004 and 2015 were reviewed for all growing children and adolescents up to 18 years of age who were treated with either PETS or PDED to correct a congenital or acquired LLD between $2 \mathrm{~cm}$ and $6 \mathrm{~cm}$ and returned for clinical follow-up at least two years postoperatively. Patients with unverified preoperative LLD or who underwent hemiepiphysiodesis procedures were excluded from the study.

PETS is typically performed by creating small stab incisions down to the femur and/or tibia, passing guidewires through the notch and medial cortex and central third on the lateral. Then, cannulated screws are measured for appropriate length and placed with planned trajectory to halt physeal growth. As patients are admitted in a day surgery setting, they are discharged within the same day and allowed to be weight-bearing as tolerated utilizing crutches and knee immobilizer. The PDED procedure typically begins with a small incision made over the area of the growth plate laterally. Dissection is carried down to the level of the growth plate, then a transverse pass is first made and radial cuts are made $5^{\circ}$ anteriorly and posteriorly. An angled curette is then introduced and metaphyseal and epiphyseal bone along with the intervening growth plate is destroyed. This same technique is applied medially, and the patient is hospitalized overnight. Postoperative protocol varies by physician, but typically involves limited ambulation or weight-bearing while wearing a knee immobilizer for two to four weeks, then return to non-contact activity after a month.

Per standard of clinical care, patients received long standing, posteroanterior (PA) hip to ankle radiographs called 'scanograms' that were in turn measured by a radiologist for LLD by directly, digitally measuring the femur length then the tibial length in conjunction. If a scanogram was unattainable, then PA radiographs demonstrating the femoral head or iliac crests were digitally measured for disparity in height. Bone age was determined by a radiologist through hand radiographs and the Greulich and Pyle method. ${ }^{16}$ Estimated growth remaining was then calculated by utilizing the bone age, gender and operative bone (femur, tibia or both) and utilizing the Green-Anderson chart. ${ }^{4} \mathrm{~A}$ total of 145 PETS and PDED procedures were identified. A total of 20 cases were excluded in which the preoperative LLD could not be verified. An additional ten cases were excluded due to insufficient follow-up, leaving 115 patients (23 PETS and 92 PDED) for analysis. Recorded patient, surgical and outcome characteristics were summarized for all patients and included: age at surgery, gender, location of epiphysiodesis, laterality, operative time, LOS, whether the patient returned to the operating room (OR) for hardware removal, postoperative complications, complaint/pain at follow-up and time to return to activity or sports. LOS analysis was only completed on patients who had an epiphysiodesis as their primary and only procedure $(n=111)$ because a concurrent procedure (such as a spine fusion or osteotomy) would confound the results. Time to return to activity was based on information obtained in clinic notes including physician recommendation or active patient participation in activities or sports. Categorical data were summarized by frequency and percentage while continuous data were summarized by mean and standard deviation (SD) or median and interquartile range (IQR, 25th to 75th percentile) when data deviated from normality.

Bone age, LLD and expected growth remaining (EGR) were calculated for each patient prior to surgery and at last follow-up. The change in each measure was calculated by subtracting the preoperative measure from the follow-up measure. Percentage change in LLD was calculated as the difference in preoperative and follow-up measurement divided by preoperative measurement. A positive percentage represents a correction or reduction in LLD such that a percentage over $100 \%$ indicates an over-correction and a negative percentage indicates an increase in LLD. The correction ratio (actual correction in LLD (change in LLD from preoperative to most-recent follow-up) divided by the expected correction (change in EGR from preoperative to most-recent follow-up)) was estimated along with a $95 \%$ confidence interval $(\mathrm{Cl})$ to assess whether correction in leg length was achieved. Values of the correction ratio close to 1 indicate successful correction.

Surgical and outcome characteristics were compared across epiphysiodesis groups. Binary and categorical data were compared using Fisher's exact test or chi-squared test, as appropriate, and continuous data were compared using Student's $t$-test or the Mann-Whitney U-test, as appropriate. Outcomes were analyzed across groups using independent samples $t$-tests and general linear modelling. A p-value less than 0.05 was considered significant. 
Table 1 Patient and treatment characteristics by treatment group

\begin{tabular}{|c|c|c|c|c|c|}
\hline \multirow[b]{2}{*}{$\begin{array}{l}\text { Characteristics at procedure } \\
\text { Age at procedure }\end{array}$} & \multicolumn{2}{|c|}{ Screw $(n=23)$} & \multicolumn{3}{|c|}{ Drilling $(n=92)$} \\
\hline & Frequency & $(\%)$ & Frequency & $(\%)$ & p-value \\
\hline$(y r s ;$ mean $\pm S D)$ & 13.3 & SD 1.62 & 12.4 & SD 1.44 & 0.03 \\
\hline Gender (\% male) & 15 & $(65)$ & 39 & $(42)$ & 0.08 \\
\hline Location & & & & & 0.39 \\
\hline Distal femur & 15 & $(65)$ & 46 & $(50)$ & \\
\hline Proximal tibia & 3 & (13) & 24 & $(26)$ & \\
\hline Combination & 5 & $(22)$ & 22 & $(24)$ & \\
\hline Side (\% right) & 13 & $(57)$ & 42 & $(46)$ & 0.36 \\
\hline $\begin{array}{l}\text { Operative time } \\
\text { (min; median (IQR); } n=108)^{*}\end{array}$ & 50 & (40 to 85 ) & 54 & (39 to 67 ) & 0.86 \\
\hline \multicolumn{5}{|l|}{ Patient age (yrs) } & p-value \\
\hline Preoperative & 13 & (12 to 14$)$ & 12 & (11 to 13 ) & 0.04 \\
\hline Most-recent measure & 16 & (14 to 17 ) & 16 & (14 to 17 ) & 0.54 \\
\hline \multicolumn{6}{|l|}{ Bone age (yrs) } \\
\hline Preoperative & 13 & (12 to 14$)$ & 12 & (11 to 14$)$ & 0.02 \\
\hline Most-recent measure & 16 & (15 to 16$)$ & 16 & (15 to 17$)$ & 0.50 \\
\hline \multicolumn{6}{|l|}{ Leg-length discrepancy (cm) } \\
\hline Preoperative & 3 & (2 to 4$)$ & 3 & (2 to 4$)$ & 0.84 \\
\hline Most-recent measure & 1 & (0 to 2$)$ & 1 & (0 to 2$)$ & 0.80 \\
\hline \multicolumn{6}{|l|}{ Expected growth remaining $(\mathrm{cm})$} \\
\hline Preoperative & 2 & (2 to 3 ) & 3 & (2 to 3 ) & 0.71 \\
\hline Most-recent measure & 0 & $(0$ to 0$)$ & 0 & (0 to 0$)$ & 0.13 \\
\hline
\end{tabular}

"the number in parentheses $(n=)$ represents the number of patients with available data for the given characteristic

IQR, interquartile range (25th percentile to 75th percentile). The p-values in the table are based on a chi-squared test, a Student's t-test, or a Mann-Whitney U-test across treatment groups, as appropriate.

At our institution PETS and PDED are performed at an approximate ratio of $1: 4$. A power analysis determined that for a 1:4 ratio of PETS to PDED patients we would require nine PETS and 36 PDED patients to achieve $80 \%$ power to detect non-inferiority using a one-sided, two-sample $t$-test across treatment groups with a non-inferiority margin of $1 \mathrm{~cm}$ in final LLD with alpha set to $5 \%$. In addition, it was determined that 12 PETS and 48 PDED patients would provide $80 \%$ power for a two-sided test of Poisson event rates to detect a one-day difference in hospital stay across treatment groups and 11 PETS and 44 PDED patients would provide $80 \%$ power for a two-sided $t$-test to detect a one-month difference in the amount of time to return to activity across groups.

A propensity score analysis was conducted to determine the presence of selection bias by treatment group. A matching algorithm was implemented based on patient gender, preoperative LLD and EGR using a nearest neighbour matching with a caliper of 0.2 . The initial cohort that met all inclusion and exclusion criteria (23 PETS and 92 PDED) was found to have a comparable distribution of propensity scores across treatment groups with negligible selection bias.

\section{Results}

A total of 115 patients who underwent epiphysiodesis in the distal femur (53\%), proximal tibia (24\%) or a combination (24\%) were analyzed with a median preoperative LLD of
$3 \mathrm{~cm}$ (IQR 2.5 to 3.8). The cohort was $47 \%$ male and the mean age at surgery was 12.6 years (SD 1.63). Median follow-up was 3.7 years (IQR 2.8 to 4.2). In all, 23 patients (20\%) underwent PETS and 92 patients underwent PDED. PDED patients were followed for a median of four years (IQR 3 to 5) compared with three years (IQR 2 to 3 ) for PETS patients $(p<0.001)$.

There were no differences in gender or location of epiphysiodesis across treatment groups (Table 1). Operative time was not different across groups (PETS: median, $50 \mathrm{~min}$ (IQR 40 to 85); PDED: 54 min (39 to 67); $p=0.86$ ). There was a significant difference in patient age at time of procedure with a mean difference of ten months (PETS: 13.3 years (SD 1.62); PDED: 12.4 years (SD 1.44); $p=0.03$ ). An average 2-cm LLD correction was achieved in both treatment groups $(95 \% \mathrm{Cl}-2.7$ to -1.5$)$ with a median LLD at most-recent measurement of $1 \mathrm{~cm}$ (IQR 0 to 2 ) in both groups (Fig. 1) (Table 2). There was no difference in mean correction ratio across groups (PETS: $1.2(95 \% \mathrm{Cl} 0.6$ to 1.8); PDED: 1.1 (95\% Cl 0.8 to 1.4); $p=0.82$ ) (Table 2).

Median hospital stay was one day in both treatment groups (IQR 0 to $1 ; p=0.91$ ) with only $7 \%$ of PDED patients and $4 \%$ of PETS patients remaining in the hospital more than one day $(p=0.93)$ (Table 2). PETS patients reported return to activity earlier at a median of 1.4 months (IQR 0.7 to 2.1); whereas PDED patients returned at a median of 2.4 months (IQR 1.7 to 3.0$)(p<0.001)$. Five PETS patients (22\%) returned to the OR: three underwent repeat epiphysiodesis and three had implant removal 


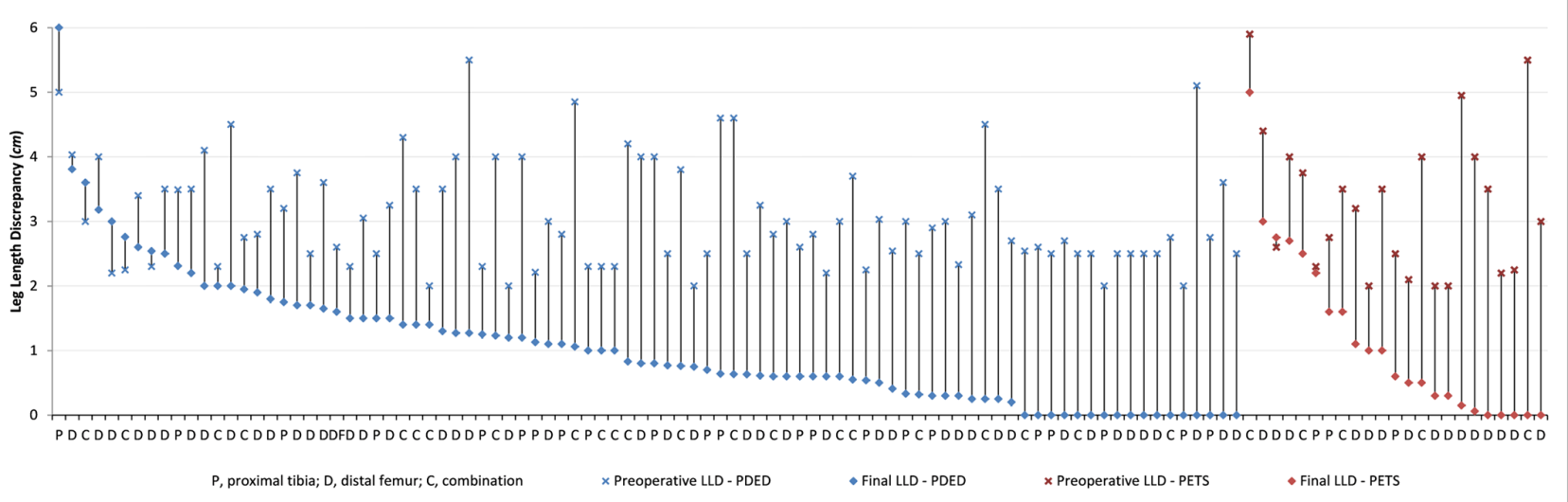

Fig. 1 Preoperative and final leg-length discrepancy (LLD) by treatment group (PDED, percutaneous drill/curettage epiphysiodesis; PETS, percutaneous epiphysiodesis using transphyseal screws).

Table 2 Outcomes by treatment group

\begin{tabular}{|c|c|c|c|c|c|}
\hline \multirow{2}{*}{ Treatment characteristics } & \multicolumn{2}{|c|}{ Screw $(n=23)$} & \multicolumn{3}{|c|}{ Drilling $(n=92)$} \\
\hline & Frequency & $(\%)$ & Frequency & $(\%)$ & p-value \\
\hline Days (median (IQR)) & 1 & (0 to 1 ) & 1 & ( 0 to 1$)$ & 0.91 \\
\hline By category & & & & & 0.93 \\
\hline Less than a day & 6 & (26) & 26 & $(30)$ & \\
\hline One day & 16 & (70) & 56 & (64) & \\
\hline More than a day & 1 & (4) & 6 & $(7)$ & \\
\hline Change in limb discrepancy & Mean change & $(95 \% \mathrm{Cl})$ & Mean change & $(95 \% \mathrm{Cl})$ & p-value \\
\hline Limb length discrepancy $(\mathrm{cm})$ & -2.1 & $(-2.7$ to -1.5$)$ & -2.0 & $(-2.3$ to -1.8$)$ & 0.71 \\
\hline Expected growth remaining $(\mathrm{cm})$ & -2.2 & $(-2.7$ to -1.8$)$ & -2.5 & $(-2.8$ to -2.2$)$ & 0.37 \\
\hline $\begin{array}{l}\text { Growth ratio (actual change in LLD/expected } \\
\text { change in LLD) }\end{array}$ & 1.2 & (0.6 to 1.8$)$ & 1.1 & (0.8 to 1.4$)$ & 0.82 \\
\hline Percentage correction LLD (\%) & 65.3 & (50.7 to 80$)$ & 65.2 & (58.4 to 71.9 ) & 0.99 \\
\hline Outcome characteristics & Frequency & (\%) & Frequency & (\%) & p-value \\
\hline Final LLD (cm; median (IQR)) & 1 & (0 to 2$)$ & 1 & (0 to 2$)$ & 0.71 \\
\hline $\begin{array}{l}\text { Time to return to activity (months; median } \\
(\text { IQR)); } n=104)^{*}\end{array}$ & 1.4 & (0.7 to 2.1$)$ & 2.4 & (1.7 to 3 ) & $<0.001$ \\
\hline Complication & 1 & (4) & 2 & (2) & 0.57 \\
\hline Return to the OR & 5 & (22) & 9 & (10) & 0.13 \\
\hline Repeat epiphysiodesis & 3 & (14) & 8 & (9) & \\
\hline Hardware removal & 3 & (13) & 0 & (0) & \\
\hline Complaints/pain at follow-up $(n=109)$ * & 6 & (26) & 18 & (21) & 0.60 \\
\hline
\end{tabular}

*the number in parentheses $(n=)$ represents the number of patients with available data for the given characteristic

IQR, interquartile range; $\mathrm{Cl}$, confidence interval; LLD, leg-length discrepancy; OR, operating room. The p-values in the table are based on a Student's $t$-test or general linear modeling analysis, as appropriate.

(one patient underwent both revision procedures). Two of the three repeat epiphysiodesis patients demonstrated under-correction on the contralateral leg, and thus required an epiphysiodesis of an additional growth plate on the surgical leg (i.e. previously operated on femur, now tibia) to further equalize. The other instance presented with unexpected rapid correction of the surgical leg requiring a contralateral epiphysiodesis in order to prevent LLD after achieving equalization. Nine PDED patients (10\%) returned to the OR; eight for repeat epiphysiodesis (six demonstrating under-correction, thus requiring a second epiphysiodesis of an additional growth plate in the initial surgical leg; two requiring epiphysiodesis of the contralateral leg to prevent over-correction) and one due to an osteochondral defect in the nonoperative leg. In all, $26 \%$ of patients within the PETS cohort complained of pain or discomfort in comparison to $21 \%$ of PDED patients.

\section{Discussion}

The PETS procedure holds potential benefits including theoretical reversibility (although more studies must be done to prove this), minimally invasive technique and rapid recovery time to baseline activity. ${ }^{13}$ For these reasons PETS has become an attractive alternative for surgeons 
who are correcting LLD. $6,9,17$ Due to the recent surge in use of the PETS technique and our centre's anecdotal experience with the operation, we hypothesized that the PETS technique would demonstrate similar efficacy in eliminating LLD, yet depict differences in rehabilitation time until return to activity, postoperative complication rate and LOS.

This study is in agreement with previous studies that have demonstrated efficacy of the different epiphysiodesis techniques. Kemnitz et $\mathrm{al}^{18}$ and Gabriel et al ${ }^{19}$ denoted the efficiency of PDED in equalizing leg lengths while showing minimal complications. Measuring true efficacy, however, is dependent on the technique chosen to estimate growth remaining of the affected physis. Monier et $\mathrm{al}^{8}$ demonstrated that the mean LLD at maturity between predicted measurements was $0.2 \mathrm{~cm}$ using the Green-Anderson method, $1.4 \mathrm{~cm}$ using the Mosely method and $-0.1 \mathrm{~cm}$ using the Paley method. ${ }^{8}$ We elected to use the Green-Anderson growth remaining charts for the current study and estimated a correction ratio (actual correction divided by expected correction) to evaluate LLD correction. Using this approach, efficacy in correcting LLD was similar for PDED and PETS methods (average correction ratio of 1.1 and 1.2 , respectively).

Each of the epiphysiodesis techniques in the present study is associated with a unique set of risks and benefits. The PDED technique provides minimal invasion, low morbidity and satisfactory leg-length equalization, yet is irreversible once performed. ${ }^{20,21}$ Due to its permanence, the technique requires accurate and precise anticipation of the patient's growth, as well as regular follow-up in order to curb any growth spurts and future inequality due to overgrowth of nonoperative leg. ${ }^{18}$ In the current study, eight PDED patients (9\%) required a second epiphysiodesis due to either under-correction or potential over-correction and subsequent LLD. When compared with the previous method of care, Phemister's open technique, the PDED technique boasts efficient equalization, and less pain. Moreover, Babu et al ${ }^{12}$ observed that PDED had a higher success rate and lower complication rate than PETS. However, PDED suffers from a potentially longer operative time, longer postoperative rehabilitation period and absence of immediate postoperative weight-bearing when compared with the PETS. ${ }^{13}$

In contrast, the PETS technique provides potential reversibility with instrumentation removal, 3,7 with the added benefits of shorter operative time, shorter postoperative rehabilitation period, shorter LOS and direct weight-bearing postoperatively with no need of knee immobilization. Campens et $\mathrm{al}^{13}$ compared the PETS technique with the Phemister and PDED technique in 80 patients, noting that the benefits of PETS (quick rehabilitation and return to activity) greatly outweighed the minor complication risks. ${ }^{13}$ The PETS cohort in this population demonstrated a lower rehabilitation time (1.4 months versus 2.4 months) in similar fashion to the literature. Moreover, Dodwell et $\mathrm{al}^{6}$ argued that complications related to the PETS technique such as revisions may be overstated, and occur less frequently than the established literature may suggest. At our institution, the current protocol allows early weight-bearing as tolerated in patients who have PETS. While the PETS technique may be reversible in the event of potential overcorrection or continued inequality, PETS techniques may present as a potential source of pain for the patient, as the screw heads may be irritative either by muscle excursion such as the vastus medialis or by prominence such as seen in the proximal tibia as observed by our institution and this series. Moreover, the reversibility of the PETS is under scrutiny as some may argue that bone resorption never fully occurs after damage to physeal cartilage ${ }^{14,15}$ Other reported complications include angular deformities and under-correction. ${ }^{22}$ Ultimately, these scenarios can lead to instrumentation removal and a costly return to the operating room; yet this analysis depicts no greater complication risk than the drill/curettage technique.

Limitations of the current study include its retrospective design, the lack of objective outcome measures and the reliance on varying physicians' subjective findings. The use of growth prediction methods including the Green-Anderson growth chart may lead to error in surgical planning and failure of leg-length equalization ${ }^{23}$ and may introduce additional bias when evaluating LLD correction. Additionally, operative time and LOS may have been influenced by surgeon-specific protocol or surgeon 'learning curve' of the PETS procedure and consequently unreflective of potential differences. The PETS procedure and protocol from a retrospective review may differ from today's practices by surgeons experienced with utilizing the PETS procedure. Moreover, measuring the rate of return to baseline activity or sports by physician approval within clinical records may be inaccurate or inconsistent by physician, thus a prospective model would benefit from objectively measuring this factor. Our data demonstrating a faster recovery time may be driven by individual rehabilitation protocols provided by differing physicians, thus may not be truly objective if some patients are given different postoperative plans.

While PETS and PDED exhibit similar efficacy with minimal differences in outcomes, one major disparity between these two procedures may be cost-effectiveness. It is likely that these procedures differ with respect to operative costs, including the cost of instrumentation and reoperation, LOS and necessary clinical follow-up. A prospective model is necessary to properly assess these costs and to observe the modern protocol in terms of LOS, operative time, rehabilitation periods and complications for both PETS and PDED techniques. 


\section{Conclusions}

This study demonstrates equal efficiency in resolving LLD between the PETS and PDED procedures with minimal operative complications. The rate of return to the OR was similar across treatment groups; however, PETS patients more commonly required a second operation due to instrumentation, while PDED patients required a second epiphysiodesis more frequently due to potential over-correction or under-correction requiring additional destroyed physes. Despite its limitations, this study can be used to design future prospective ventures that quantify patient-reported outcomes and compare cost effectiveness of these two surgical techniques.

Received 07 March 2018; accepted after revision 17 August 2018.

\section{COMPLIANCE WITH ETHICAL STANDARDS}

\section{FUNDING STATEMENT}

No benefits in any form have been received or will be received from a commercial party related directly or indirectly to the subject of this article.

\section{OA LICENCE TEXT}

This article is distributed under the terms of the Creative Commons Attribution-Non Commercial 4.0 International (CC BY-NC 4.0) licence (https://creativecommons. org/licenses/by-nc/4.0/) which permits non-commercial use, reproduction and distribution of the work without further permission provided the original work is attributed.

\section{ETHICAL STATEMENT}

Ethical approval: The study was approved by the Institutional Review Board. All procedures performed in studies involving human participants were in accordance with the ethical standards of the institutional and/or national research committee and with the 1964 Helsinki declaration and its later amendments or comparable ethical standards.

Informed consent: A waiver of informed consent was granted due to the retrospective nature of the study.

\section{ICMJE CONFLICT OF INTEREST STATEMENT}

All authors declare they have no conflicts of interest with regard to this study.

\section{REFERENCES}

1. Phemister DB. Operative arrestment of longitudinal growth of bones in the treatment of deformities. J Bone Joint Surg [Am] 1933;15-A:1-15.

2. Canale ST, Christian CA. Techniques for epiphysiodesis about the knee. Clin Orthop Relat Res 1990;255:81-85.

3. Métaizeau J-P, Wong-Chung J, Bertrand H, Pasquier P. Percutaneous epiphysiodesis using transphyseal screws (PETS). J Pediatr Orthop 1998;18:363-369.

4. Anderson M, Green WT, Messner MB. Growth and predictions of growth in the lower extremities. J Bone Joint Surg [Am] 1963;45-A:1-14.
5. Haas SL. Restriction of bone growth by pins through the epiphyseal cartilaginous plate. J Bone Joint Surg [Am] 1950;32-A:338-343.

6. Dodwell ER, Garner MR, Bixby E, et al. Percutaneous epiphysiodesis using transphyseal screws: a case series demonstrating high efficacy. HSS J 2017;13: 255-262.

7. Khoury JG, Tavares JO, McConnell S, Zeiders G, Sanders JO. Results of screw epiphysiodesis for the treatment of limb length discrepancy and angular deformity. J Pediatr Orthop 2007;27:623-628.

8. Monier BC, Aronsson DD, Sun M. Percutaneous epiphysiodesis using transphyseal screws for limb-length discrepancies: high variability among growth predictor models. J Child Orthop 2015;9:403-410.

9. Song MH, Choi ES, Park MS, et al. Percutaneous epiphysiodesis using transphyseal screws in the management of leg length discrepancy: optimal operation timing and techniques to avoid complications. J Pediatr Orthop 2015;35:89-93.

10. Niedzielski K, Flont P, Domżalski M, Lipczyk Z, Malecki K. Lower limb equalization with percutaneus epiphysiodesis of the knee joint area. Acta Orthop Belg 2016;82:843-849.

11. Ramseier LE, Sukthankar A, Exner GU. Minimal invasive epiphysiodesis using a modified "Canale"-technique for correction of angular deformities and limb leg length discrepancies. J Child Orthop 2009;3:33-37.

12. Babu LV, Evans O, Sankar A, et al. Epiphysiodesis for limb length discrepancy: a comparison of two methods. Strateg Trauma Limb Reconstr 2014;9:1-3.

13. Campens C, Mousny M, Docquier PL. Comparison of three surgical epiphysiodesis techniques for the treatment of lower limb length discrepancy. Acta Orthop Belg 2010;76:226-232.

14. Stevens P. Guided growth: 1933 to the present. Strateg Trauma Limb Reconstr 2006;:1:29-35.

15. Vogt B, Schiedel F, Rödl R. Guided growth in children and adolescents. Correction of leg length discrepancies and leg axis deformities. Orthopade 2014;43:267-284.

16. Greulich WW, Pyle SI, Todd TW. Radiographic atlas of skeletal development of the hand and wrist. Stanford: Stanford University Press, 1959.

17. Nouth F, Kuo LA. Percutaneous epiphysiodesis using transphyseal screws (PETS): prospective case study and review. J Pediatr Orthop 2004;24:721-725.

18. Kemnitz S, Moens P, Fabry G. Percutaneous epiphysiodesis for leg length discrepancy. J Pediatr Orthop B 2003;12:69-71.

19. Gabriel KR, Crawford AH, Roy DR, True MS, Sauntry S. Percutaneous epiphyseodesis. J Pediatr Orthop 1994;14:358-362.

20. Ogilvie JW, King K. Epiphysiodesis: two-year clinical results using a new technique. J Pediatr Orthop 1990;10:809-811.

21. Horton GA, Olney BW. Epiphysiodesis of the lower extremity: results of the percutaneous technique. J Pediatr Orthop 1996;16:180-182.

22. Ilharreborde B, Gaumetou $\mathbf{E}$, Souchet $\mathbf{P}$, et al. Efficacy and late complications of percutaneous epiphysiodesis with transphyseal screws. J Bone Joint Surg [Br] 2012;94-B:270-275.

23. Blair VP III, Walker SJ, Sheridan JJ, Schoenecker PL. Epiphysiodesis: a problem of timing. J Pediatr Orthop 1982;2:281-284. 